\title{
Integrability of trigonometric series with coefficients from the class $\mathrm{K}$
}

\author{
XHEVAT Z. KRASNIQI
}

\section{ABSTRACT.}

In this paper we deal with a class of sine and cosine trigonometric series. We have imposed some conditions on their coefficients so that their sums will be integrable with the exception of two points. In fact, some estimates of the integrals of the absolute values of their sums, expressed in terms of the coefficients, are obtain extending some results of a paper Telyakovskiř, S. A., Localizing the conditions of integrability of trigonometric series, Trudy Mat. Inst. Steklov., 210 (1995), 264-273 (Russian).

\section{REFERENCES}

[1] Garret, J. W., Rees, C. S. and Stanojević, Č. V., $L^{1}$-convergence of Fourier series with coefficients of bounded variation, Proc. Amer. Math. Soc., 80 (1980), No. 3, 423-430

[2] Kano, T., Coefficients of some trigonometric series, J. Fac. Sci. Shinshu Univ., 3 (1968), 153-162

[3] Kaur, K. and Bhatia, S. S., Integrability and $L^{1}$-convergence of Rees-Stanojevic sums with generalized semi-convex coefficients, Int. J. Math. and Math. Sci., 30 (2002), No. 11, 645-650

[4] Kaur, K., Bhatia, S. S. and Ram, B., Integrability and $L^{1}$-convergence of modified sine sums, Georgian Math. J., 11 (2004), No. 1, $99-104$

[5] Kolmogorov, A. N., Sur l'ordre de grandeur des coefficients de la série de Fourier- Lebesgue, Bulletin de l'Academie Polonaise, (1923), 83-86

[6] Krasniqi, Xh. Z., Integrability of cosine trigonometric series with coefficients of bounded variation of order p, Appl. Math. E-Notes, 11 (2011), 61-66

[7] Krasniqi, Xh. Z., On the first derivative of the sums of trigonometric series with quasi-convex coefficients of higher order, Acta Comment. Univ. Tartu. Math., 14 (2010), 1-11

[8] Krasniqi, Xh. Z., Integrability of double cosine trigonometric series with coefficients of bounded variation of second order, Comment. Math. Prace Mat., 51 (2011), No. 2, 125-139

[9] Krasniqi, Xh. Z., Integrability of cosine trigonometric series with semi-convex coefficients, Opuscula Math., 32 (2012), No. 3, 521-528

[10] Telyakovskiĭ, S. A., Localizing the conditions of integrability of trigonometric series, Trudy Mat. Inst. Steklov., 210 (1995), 264-273 (Russian)

DEPARTMENT OF MATHEMATICS AND INFORMATICS

UNIVERSITY OF PRISHTINA

FACULTY OF EDUCATION

Avenue "Mother TheresA" 5, 10000 Prishtinë, KosovË

E-mail address: xhevat.krasniqieuni-pr.edu 